\title{
SUBNORMAL GENERALIZED HAUSDORFF OPERATORS
}

\author{
B. K. GHOSH, B. E. RHOADES AND D. TRUTT
}

\begin{abstract}
One type of generalized Hausdorff matrix is the lower triangular matrix with entries $h_{n k}=\left(\begin{array}{c}n+\alpha \\ n-k\end{array}\right) \Delta^{n-k_{k}}$, where $\Delta \nu_{n}=\nu_{n}-\nu_{n+1}, \nu_{n}=$ $\int_{0}^{1} t^{n+\alpha} d \beta(t)$ for some $\beta(t)$ of bounded variation on $[0,1]$ and for some $\alpha>0$. The matrix $H_{\alpha}$ generated by $\nu_{n}=(n+\alpha+1)^{-1}$ is shown to be a subnormal operator on $l^{2}$ if $\alpha$ is a nonnegative integer.
\end{abstract}

1. Introduction and summary. One type of generalized Hausdorff matrix is the lower triangular matrix with entries

$$
h_{n k}=\left(\begin{array}{c}
n+\alpha \\
n-k
\end{array}\right) \Delta^{n-k} v_{k}
$$

where

$$
\Delta \nu_{n}=\nu_{n}-\nu_{n+1}, \quad \nu_{n}=\int_{0}^{1} t^{n+\alpha} d \beta(t)
$$

for some $\beta(t)$ of bounded variation on [0,1] and for some $\alpha \geqslant 0$ (see [4]). Let $H_{\alpha}$ denote the generalized Hausdorff matrix generated by $\nu_{n}=\int_{0}^{1} t^{n+\alpha} d t$. Then $h_{n k}=(n+\alpha+1)^{-1}$, so that $H_{\alpha}$ is a factorable matrix. (A lower triangular matrix $A=\left(a_{n k}\right)$ is called factorable if $a_{n k}=b_{n} c_{k}$ when $k \leqslant n$.) By [7, Theorem 2], any matrix which formally commutes with $H_{\alpha}$ must be triangular and, hence, by the proof of Theorem 198 in [3], must also be a generalized Hausdorff matrix. Thus, the operators on $l^{2}$ which commute with $H_{\alpha}$ are all generalized Hausdorff matrices. (For each $\alpha$, the commutant of $H_{\alpha}$ is a closed algebra of operators. For $a \neq \beta$, the only operators which commute with both $H_{\alpha}$ and $H_{\beta}$ are the zero operator and the identity operator.)

Our main result is that, for every nonnegative integer $\alpha, H_{\alpha}$ is a subnormal operator on $l^{2}$ and hence, by Yoshino's theorem [8] and the comments above, so is any generalized Hausdorff matrix which maps $l^{2}$ into $l^{2}$ and commutes with $H_{\alpha}$. We explicitly describe a Borel probability measure $\mu_{\alpha}$ on the closed unit disk (actually on the circles $|z-n /(n+1)|=1 /(n+1)$ ) such that $I-H_{\alpha}$ is unitarily equivalent to the operator $f(z) \rightarrow z f(z)$ acting on $H^{2}\left(\mu_{\alpha}\right)$, the closure of the polynomials in the metric of $L^{2}\left(\mu_{\alpha}\right)$. For $\alpha=0$, this result was obtained in [5], [6]. For $\alpha$ a positive integer, the result is new. We are unable to determine whether $H_{\alpha}$ is subnormal for nonnegative noninteger values of $\alpha$. Our approach relies on the methods and results of [1], [5], [6].

Received by the editors September 30, 1976.

AMS (MOS) subject classifications (1970). Primary 33A15, 33A30, 33A65, 40G05, 47B20.

- American Mathematical Society 1977 
2. Let $\alpha \geqslant 0$. We consider $H_{\alpha}$ as a linear transformation on $l^{2}$ or, equivalently, on the Hardy space $H^{2}$ of functions $f(z)=\sum a_{n} z^{n}$ analytic for $|z|<1$ with $\Sigma\left|a_{n}\right|^{2}<\infty$.

LEMMA 1. For each $\alpha \geqslant 0, H_{\alpha}$ is a bounded linear operator on $l^{2}$ with $\left\|H_{\alpha}\right\|=2$. The point spectrum of $H_{\alpha}^{*}$ is the disk $\{\lambda:|1-\lambda|<1\}$. Each $\lambda$ in this disk is a simple eigenvalue with corresponding eigenfunction $\phi_{\lambda}$, where $\phi_{\lambda}(0)=1$ and $\phi_{\lambda}(n)=\prod_{j=1}^{n}\left(1-\lambda^{-1}(j+\alpha)^{-1}\right), n=1,2,3, \ldots$

Proof. The first statement is proved in $[4$, p. 177]. The proof of the second statement is the same as the proof of Theorem 2 in [1].

If $f$ belongs to $l^{2}$, define $F$ by $F(z)=\left\langle f, \phi_{\bar{z}}\right\rangle_{l^{2}}$, for $|z|<1$. The correspondence $f \leftrightarrow F$ is $1-1$ since the elements $\phi_{z}$, for $|z|<1$, span a dense subset of $l^{2}$. (For $\lambda=(\alpha+k)^{-1}, k=1,2,3, \ldots, \phi_{\lambda}(k)=0$ for $n \geqslant k$ and $\phi_{\lambda}(n) \neq 0$ for $n \leqslant k-1$.) Let $\mathcal{H}_{\alpha}$ denote all such functions $F$, and define $\|F\|=\|f\|_{l^{2} \text {. }}$ Then $\mathcal{H}_{\alpha}$ is a Hilbert space of functions analytic for $|z|<1$. (The analyticity follows since the reproducing kernel function for $\mathcal{H}_{\alpha}, k(w, z)=\left\langle\phi_{\bar{w}}, \phi_{\bar{z}}\right\rangle_{H^{2}}$ is the generalized hypergeometric function

$$
{ }_{3} F_{2}(-\bar{w} /(1-\bar{w})+\alpha,-z /(1-z)+\alpha, 1 ; \alpha+1, \alpha+1,1),
$$

which is analytic for $|z|<1$.)

LEMMA 2. The operator $I-H_{\alpha}$ in $l^{2}$ is unitarily equivalent to the operator $f(z) \rightarrow z f(z)$ in $\mathcal{H}_{\alpha}$. The functions $1,(z-1)^{-n}(z-\alpha /(1+\alpha)) \ldots(z-(n$ $+\alpha-1) /(n+\alpha))$, for $n \geqslant 1$, are an orthonormal basis for $\mathcal{H}_{\alpha}$.

Proof. Lemma 2 follows from the proofs in [6, p. 216].

The mapping $z /(1-z)$ takes the disk $|z|<1$ onto the half-plane $\operatorname{Re} w>-\frac{1}{2}$. The inverse map is $w \rightarrow w /(1+w)$. Let $\mathscr{K}_{\alpha}$ denote the set of functions $G$ of the form $G(z)=F(z /(1+z))$ for some $F$ in $\mathcal{H}_{\alpha}, \operatorname{Re} z>$ $-\frac{1}{2}$. Define a norm in $\mathcal{K}_{\alpha}$ by $\|G\|=\|F\|_{\mathcal{K}_{\alpha}}$. Then $\mathcal{K}_{\alpha}$ is a Hilbert space of functions analytic for $\operatorname{Re} z>-\frac{1}{2}$ with orthonormal basis $\psi_{0}=1$,

$$
\psi_{n}(z)=(-1)^{n} \frac{\Gamma(\alpha+1)}{\Gamma(n+\alpha+1)}(z-\alpha)(z-\alpha-1) \ldots(z-\alpha-n+1) .
$$

Since

$$
\psi_{n}(z)=\frac{\Gamma(n+1) \Gamma(\alpha+1)}{\Gamma(n+\alpha+1)} \phi_{n}(z-\alpha)
$$

where

$$
\phi_{n}(z)=(n !)^{-1} z(z-1) \ldots(z-n+1),
$$

it follows from [6, p. 218] that for $0<a, b<2, a^{z}$ and $b^{z}$ belong to $\mathcal{K}_{\alpha}$ and

$$
\begin{aligned}
\left\langle a^{z}, b^{z}\right\rangle_{\mathcal{K}_{\alpha}} & =(a b)^{\alpha} \sum_{0}^{\infty} \frac{\Gamma^{2}(n+\alpha+1)}{\Gamma^{2}(n+1) \Gamma^{2}(\alpha+1)} \\
& =(a b)^{h-1} F(h, h ; 1 ;(1-a)(1-b)),
\end{aligned}
$$


where $h=\alpha+1$ and

$$
F(h, h ; 1 ; c)=\sum_{j=0}^{\infty} \frac{\Gamma^{2}(h+j)}{\Gamma^{2}(h)(j !)^{2}} c^{j}
$$

is the hypergeometric function. This result, together with Theorem 1 below, will show that the norm in $\mathcal{K}_{\alpha}$ is given by integration with respect to a measure on the half-plane $\operatorname{Re} w \geqslant-\frac{1}{2}$. (We will retain the notation $h=\alpha+$ 1 throughout.)

LEMMA 3. For all real numbers $a, b$ and nonnegative integers $x$,

$$
\sum_{j=0}^{m}(-1)^{j} \frac{\Gamma(a+1) \Gamma(b+j)}{j !(m-j) ! \Gamma(b) \Gamma(a-m+j+1)}=(-1)^{m} \frac{\Gamma(b-a+m)}{m ! \Gamma(b-a)},
$$

where we interpret $1 / \Gamma(-n+1)=0$ and $\Gamma(-c+n) / \Gamma(-c)=(-c)(-c+$ 1) $\ldots(-c+n-1)$ for all positive integers $n$.

Proof. The identity follows if one equates the coefficients of $t^{m}$ in the binomial expansions of both sides of the identity $(1-t)^{a}(1-t)^{-b}=(1-$ $t)^{-(b-a)}$.

COROLLARY. If $h>k$ are nonnegative integers, then

$$
\sum_{j=0}^{h-k-1}(-1)^{j} \frac{\Gamma(h+k+j)}{j !(k+j) ! \Gamma(h-j-k)}=(-1)^{h-k-1} \frac{\Gamma(h+k)}{k ! \Gamma(h-k)} .
$$

Proof. Let $a=h-1, b=h+k$ and $n=h-k-1$ in Lemma 3. The result follows.

LEMMA 4. For $h$ a positive integer and $|c|<1$, we have the identity

$$
F(h, h ; 1 ; c)=(1-c)^{-h} \sum_{k=0}^{h-1} \frac{(-1)^{h-k-1} \Gamma(h+k)}{(k !)^{2} \Gamma(h-k)}(1-c)^{-k} .
$$

Proof. Using formulas (4) on p. 57 and (22) on p. 64 of [2], and the identity $\Gamma(h) \Gamma(1-h) / \Gamma(1-h+j)=(-1)^{j} \Gamma(h-j)$, we get

$$
\begin{aligned}
F(h, h ; 1 ; c) & =(1-c)^{-h} F(h, 1-h ; 1 ; c /(c-1)) \\
& =(1-c)^{-h} \sum_{j=0}^{h-1} \frac{\Gamma(h+j)}{(j !)^{2} \Gamma(h-j)}\left(\frac{c}{1-c}\right)^{j} .
\end{aligned}
$$

Expand $(c /(1-c))^{j}=(1 /(1-c)-1)^{j}$ by the binomial theorem and set $k=j-i$. The result then follows from the Corollary.

THEOREM 1. For each positive integer $h$ and all $0<a, b<2$,

$$
(a b)^{h-1} F(h, h, 1 ;(1-a)(1-b))=\int_{-\infty}^{\infty} \int_{-\infty}^{\infty} a^{z} b^{\bar{z}} d \beta_{h}(x, y),
$$

where $\beta_{h}(x, y)$ is a joint probability measure defined by 


$$
d \beta_{h}(x, y)=\frac{|\Gamma(x+1+i y)|^{4} d y d \mu(x)}{2 \pi \Gamma^{2}(h) \Gamma(2 x+2)|\Gamma(x-h+2+i y)|^{2}},
$$

where $\mu(x)=1$ if $x=(n-1) / 2$ for some $n=0,1,2,3, \ldots$, and $\mu(x)=0$ otherwise.

Proof. By Lemma 4,

$$
\begin{aligned}
&(a b)^{h-1} F(h, h, 1 ;(1-a)(1-b)) \\
&=(a b)^{h-1} \sum_{k=0}^{h-1} \frac{(-1)^{h-k-1} \Gamma(h+k)}{(k !)^{2} \Gamma(h-k)}(a+b-a b)^{-(h+k)} .
\end{aligned}
$$

By Theorem 1 in [5],

$$
(a+b-a b)^{-(h+k)}=\sum \int_{-\infty}^{\infty} \frac{|\Gamma(x+h+k+i y)|^{2} a^{z} b^{\bar{z}}}{2 \pi \Gamma(2 x+h+k+1) \Gamma(h+k)} d y,
$$

where the sum is over all $x$ of the form $x=(n-h-k) / 2$, for $n=$ $0,1,2, \ldots$ It follows, after a change of variables $x^{\prime}=x+h-1$, that

$$
\begin{aligned}
(a b)^{h-1} F & (h, h, 1 ;(1-a)(1-b)) \\
= & \sum_{k=0}^{h-1} \sum \int_{-\infty}^{\infty} \frac{(-1)^{h-k-1}|\Gamma(x+k+1+i y)|^{2} a^{z} b^{\bar{z}} d y}{2 \pi(k !)^{2} \Gamma(2 x-h+k+3) \Gamma(h-k)},
\end{aligned}
$$

where the inner sum is over all $x$ of the form $x=\frac{1}{2}(n+h-k-2)$, for $n=0,1,2, \ldots$ Thus,

$$
\begin{aligned}
(a b)^{h-1} F(h, h, 1 ;(1-a)(1-b)) \\
=\frac{1}{2 \pi} \sum \int_{-\infty}^{\infty} A_{h}(x, y) a^{z} b^{\bar{z}} d y \\
\quad+\frac{1}{2 \pi} \sum \int_{-\infty}^{\infty} B_{h}(x, y) a^{z} b^{\bar{z}} d y,
\end{aligned}
$$

where the first sum is over $x$ in $\{-1 / 2,0,1 / 2, \ldots, h / 2-3 / 2\}$, the second sum is over $x$ in $\{h / 2-1 / 2, h / 2, \ldots\}$ and

$$
\begin{aligned}
& A_{h}(x, y)=\sum_{k=h-2-2 x}^{h-1} \frac{(-1)^{h-k-1}|\Gamma(x+k+1+i y)|^{2}}{(k !)^{2} \Gamma(2 x-h+k+3) \Gamma(h-k)}, \\
& B_{h}(x, y)=\sum_{k=0}^{h-1} \frac{(-1)^{h-k-1}|\Gamma(x+k+1+i y)|^{2}}{(k !)^{2} \Gamma(2 x-h+k+3) \Gamma(h-k)} .
\end{aligned}
$$

Now, Saalschütz's Theorem $[2$, p. 188] states that for $m=0,1,2, \ldots$,

$$
\begin{aligned}
\sum_{j=0}^{m} \frac{(-m)_{j}}{\Gamma(\alpha+j) \Gamma(\beta+j)} & \\
= & \frac{\Gamma(\alpha) \Gamma(\beta) \Gamma(c-\alpha+m) \Gamma(c-\beta+m)}{\Gamma(c-\alpha) \Gamma(c-\beta)(c)_{m}(c-\alpha-\beta)_{m}}
\end{aligned}
$$


where $(c)_{j}=c(c+1) \ldots(c+j-1)$ for $j \geqslant 1$, and $(c)_{0}=1$. Putting $m=h$ $-1, \alpha=x+1+i y, \beta=x+1-i y, c=2 x-h+3$, and noting $\Gamma(h-k)$ $=(-1)^{k} \Gamma(h) /(-h+1)_{k}$, it follows that

$$
B_{h}(x, y)=|\Gamma(x+1+i y)|^{4}\left\{\Gamma^{2}(h) \Gamma(2 x+2)|\Gamma(x-h+2+i y)|^{2}\right\}^{-1} \text {. }
$$

To simplify $A_{h}(x, y)$, put $k^{\prime}=k-h+2+2 x$, and then let $m=2 x+1$, $\alpha=h-1-x+i y, \beta=h-1-x-i y, c=h-2 x-1$ in Saalschütz's Theorem to get

$$
\begin{aligned}
A_{h}(x, y)= & |\Gamma(x+1+i y)|^{2}|\Gamma(h-1-x+i y)|^{2} \\
& \times\left\{\Gamma^{2}(h) \Gamma(2 x+2)|\Gamma(-x+i y)|^{2}\right\}^{-1} .
\end{aligned}
$$

It follows that $A_{h}(x, y)=B_{h}(x, y)$, which establishes the theorem except for the verification that $\beta_{h}$ is a probability measure. But this follows from the observation that

$$
\sum \int_{-\infty}^{\infty} \frac{1}{2 \pi} B_{h}(x, y) d y=F(h, h, 1 ; 0)=1 \text { and } B_{h}(x, y)>0 .
$$

THEOREM 2. If $\alpha=0,1,2,3, \ldots$, then the operator $I-H_{\alpha}$ on $l^{2}$ is subnormal.

Proof. The proof is essentially the same as the proof of Theorem 2 in [5].

REMARK. The formula for $\beta_{h}(x, y)$ in Theorem 1 defines a positive measure whenever $h \geqslant 1$. However, the functions $\psi_{n}(z)$ which form an orthonormal basis for $\mathcal{K}_{\alpha}$ are neither orthogonal nor of norm 1 in the metric of $L^{2}\left(\alpha_{h}\right)$ unless $h$ is a positive integer. Thus, the most natural candidate for a measure which determines the norm in $\mathscr{K}_{\alpha}$, for noninteger $\alpha$, fails.

The authors gratefully acknowledge helpful correspondence with Professor Louis de Branges.

\section{REFERENCES}

1. A. Brown, P. R. Halmos and A. L. Shields, Cesaro operators, Acta. Sci. Math. (Szeged) 26 (1965), 125-137.

2. A. Erdélyi, W. Magnus, F. Oberhettinger and F. G. Tricomi, Higher transcendental functions, Vol. 1, McGraw-Hill, New York, 1953.

3. G. H. Hardy, Divergent series, Oxford Univ. Press, 1949.

4. A. Jakimovski, B. E. Rhoades and J. Tzimbalario, Hausdorff matrices as bounded operators over IP, Math. Z. 138 (1974), 173-181.

5. E. Kay, H. Soul and D. Trutt, Some subnormal operators and hypergeometric kernel functions, J. Math. Anal. Appl. 53 (1976), 237-242.

6. T. L. Kriete and D. Trutt, The Cesaro operator in $l^{2}$ is subnormal, Amer. J. Math. 93 (1971), 215-225.

7. B. E. Rhoades, Commutants of some Hausdorff matrices, Pacific J. Math. 43 (1972), 715-719; 49 (1973), 617-619.

8. B. E. Rhoades and A. Wilansky, Some commutants in B $(c)$ which are almost matrices, Pacific J. Math. 49 (1973), 211-217.

9. T. Yoshino, Subnormal operators with a cyclic vector, Tohoku Math. J. 21 (1969), 47-55.

Department of Mathematics, Indiana University, Bloomington, Indiana 47401

Department of Mathematics, Lehigh University, Bethlehem, Pennsylvania 18015 